\title{
Online course Geometrical Optics for undergraduate students
}

\author{
Alexey Bakholdin, Anna Voznesenskaya, Galina \\ Romanova, Tatiana Ivanova, Nadezhda Tolstoba, et al.
}

Alexey Bakholdin, Anna Voznesenskaya, Galina Romanova, Tatiana Ivanova, Nadezhda Tolstoba, Kseniia Ezhova, Aleksei Garshin, Oleg Trifonov, Dmitry Sazonenko, Alisa Ekimenkova, "Online course Geometrical Optics for undergraduate students," Proc. SPIE 10452, 14th Conference on Education and Training in Optics and Photonics: ETOP 2017, 104521S (16 August 2017); doi: 10.1117/12.2266491

SPIE Event: 14th Conference on Education and Training in Optics and Photonics, ETOP 2017, 2017, Hangzhou, China 


\title{
On-line course "Geometrical optics" for undergraduate students
}

\author{
Alexey Bakholdin, Anna Voznesenskaya, Galina Romanova*, Tatiana Ivanova, Nadezhda Tolstoba, \\ Kseniia Ezhova, Aleksei Garshin, Oleg Trifonov, Dmitry Sazonenko, Alisa Ekimenkova \\ ITMO University \\ 49 Kronverksky av., St. Petersburg, 197101, Russian Federation
}

\begin{abstract}
The paper is devoted to the description of the on-line course "Geometrical Optics" placed on the national open-education platform. The course is purposed mainly for undergraduate students in optics and related fields. We discuss key features of the on-line form of this course, the issues of its realization and learning outcomes' evaluation.
\end{abstract}

Keywords: e-learning, geometrical optics, optical education, learning outcomes evaluation

\section{INTRODUCTION}

Recently there is a growing interest to the e-learning courses. The e-learning allows expanding knowledge and developing practical skills in the professional area and related fields distantly. ITMO University [1] uses the distant forms of learning as a part of many courses that are studied in the university. ITMO University students use the Center of Distant Education (CDE) to pass some tests, see the lectures and other materials downloaded to the system by teachers [2]. To do the professional education more distributed and available to the more audience the federal educational program was started. There is a national educational resource of e-learning in various fields of knowledge used by the universities of the country [3]. On the site one can find many courses that start several times a year, have different forms and presented by different universities of the country. Many courses can give one ECTS that can be used later in education courses.

Education in optics and optical engineering is a core for many industrial applications deal with research and hightechnology projects. The on-line course "Geometrical Optics" is developed to be a basis of the undergraduate optical education.

\section{COURSE TOPICS}

The discussed course was developed by Applied and Computer Optics Department of ITMO University [4]. The course is aimed to build knowledge in fundamental laws of geometrical optics, their relation to the physical laws. It also considers operation of optical components and simple optical systems. The course demonstrates the place of optics as a whole among other related courses. It discusses using the geometrical optics laws at the development of optical devices and instruments and also for the understanding of the working principles of various optical devices.

"Geometrical optics" includes theoretical description that is necessary for initial modeling and performance analysis of optical systems, and is supported with a considerable amount of practical tasks for the topics as well as virtual lab devoted to the ray tracing and image modeling.

The following topics are included into the on-line course:

- $\quad$ Light fields and waves

- Energy and light values

- Geometrical optics laws

- Paraxial optics and ray tracing

*romanova_g_e@ @ mail.ru; phone +7 812 595-4165; http://aco.ifmo.ru

14th Conference on Education and Training in Optics and Photonics: ETOP 2017, edited by Xu Liu,

Xi-Cheng Zhang, Proc. of SPIE Vol. 10452, 104521S · @ 2017 ICO, IEEE, OSA, SPIE

CCC code: $0277-786 \mathrm{X} / 17 / \$ 18 \cdot$ doi: $10.1117 / 12.2266491$

Proc. of SPIE Vol. 10452 104521S-1 
- $\quad$ Types and performance of optical systems

- $\quad$ Matrix optics

- Real optical surfaces and systems

- The limitations of light beams in optical systems

- Optical components

The course in fact is necessary to those students who plan to become master students in the field of optics and photonics, but their basic education is not related to these areas. As a rule a typical student should have basic ideas about physical optics, particularly, photometry, interference, diffraction, polarization and other physical phenomenon. Since a set of topics include some discussion of the phenomenon, but not in details, we need to give connections to the other areas to form the whole picture about the area of knowledge.

Every topic assumes one-week learning; the 10th week is purposed for the final e-exam.

\section{TOPICS OVERVIEW}

The first section of the course in fact is an introductory part of the course. This part is a standard for such type of courses. It explains the Maxwell's equations, discusses the special properties of the light waves compared to all other electromagnetic waves, and repeats the information about the optical bandwidth and its sub-bands.

The fig. 1 shows the one picture from the course slides. The form of presentation is also standard, because the first part should provide the clear and understandable basis for the other courses. Of cause, any interesting facts, phenomenon demonstrations, etc. can make for the deeper interest, but if we have too much such an entertainment content in the course it makes it not so clear: the entertainment information can hide the main theoretical ideas compared to the bright contents.

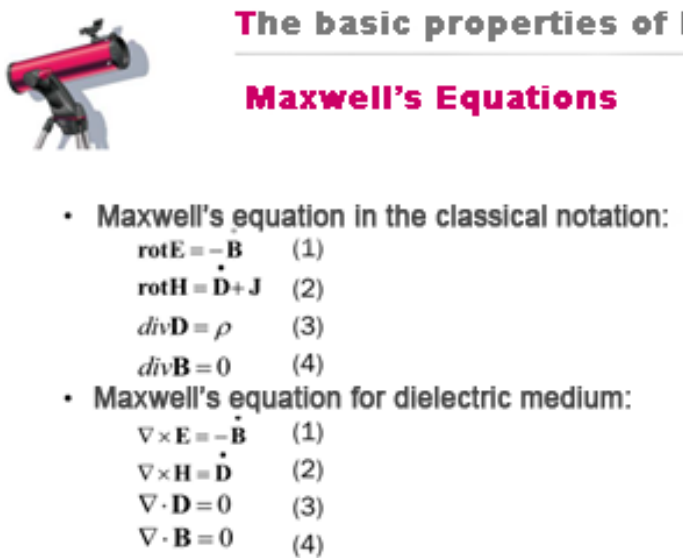

Fig.1 The example of the slide from the first part of the course "Geometrical Optics". Maxwell's equations

The second part of the course is about the energy and energetic relations. This part is obligatory and also standard, because development of any optical units, instruments and devices requires taking into account the energy transfer through the optical system. Here we discuss the simple source models that can be useful for rough evaluation of the system characteristics. We also consider the units for the energy and light values and give some examples of them for various objects to give some connections of this theoretical part to the real world.

The third part considers geometrical optics laws and invariants. This unit discusses the main laws and how they originate from the physical theory. The same part discusses simple examples of using the laws in our real life and where we can observe it, we have also included useful expressions of how light propagates through the borders between different optical media, the Fresnel equations and some information about reducing the losses due to reflection by the border.

The fourth part describes the paraxial optics and ray tracing. This part discusses the ideal optical systems. First of all, when and why we need and could consider the optical system as an ideal. That unit considers the derivation of the 
paraxial optics laws (Gaussian optics) and its practical application. For this part of the course we have a large base of tests and practical exercises.

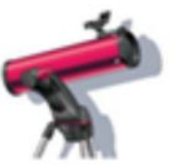

\section{Frenel's formulas}

\section{Frenel's formulas}

- There are Frenel's formulas for the passed $T_{\|}, T_{\perp}$ and refracted $R_{\|}, R_{\perp}$ waves

$$
\left\{\begin{array}{l}
T_{\|}=\frac{2 n \cos \varepsilon}{n^{\prime} \cos \varepsilon+n \cos \varepsilon^{\prime}} A_{\|} \\
T_{\perp}=\frac{2 n \cos \varepsilon}{n^{\prime} \cos \varepsilon^{\prime}+n \cos \varepsilon} A_{\perp}
\end{array}\right.
$$

Or

$$
\left\{\begin{array}{l}
T_{\|}=\frac{2 \sin \varepsilon^{\prime} \cos \varepsilon}{\sin \left(\varepsilon+\varepsilon^{\prime}\right) \cos \left(\varepsilon-\varepsilon^{\prime}\right)} A_{\|} \\
T_{\perp}=\frac{2 \sin \varepsilon^{\prime} \cos \varepsilon}{\sin \left(\varepsilon+\varepsilon^{\prime}\right)} A_{\perp}
\end{array}\right.
$$

$$
\left\{\begin{array}{l}
R_{\|}=\frac{n^{\prime} \cos \varepsilon-n \cos \varepsilon^{\prime}}{n^{\prime} \cos \varepsilon+n \cos \varepsilon^{\prime}} A_{\|} \\
R_{\perp}=\frac{n \cos \varepsilon-n^{\prime} \cos \varepsilon^{\prime}}{n \cos \varepsilon+n^{\prime} \cos \varepsilon^{\prime}} A_{\perp}
\end{array}\right.
$$

$$
\left\{\begin{array}{l}
R_{\|}=\frac{\operatorname{tg}\left(\varepsilon-\varepsilon^{\prime}\right)}{\operatorname{tg}\left(\varepsilon+\varepsilon^{\prime}\right)} A_{\|} \\
R_{\perp}=-\frac{\sin \left(\varepsilon-\varepsilon^{\prime}\right)}{\sin \left(\varepsilon+\varepsilon^{\prime}\right)} A_{\perp}
\end{array}\right.
$$

Fig.2 The example of the slide from the third part of the course "Geometrical Optics". Fresnel formulas

The fifth part includes some information about the optical systems' classification based on the object and image position. According to that classification we have four types of the system, depending on that we can describe the imaging properties of the system using different characteristics. Even the same characteristic, for example, aperture or field would mean different sense (and meaning) for telescopic system, microscope, photo- or projection system. This unit also discusses the sign condition for the radii, thickness and distances if we want to describe the system, for example, to set it to the optical design software. On the figure one can see the example of the slide to that part of the course.

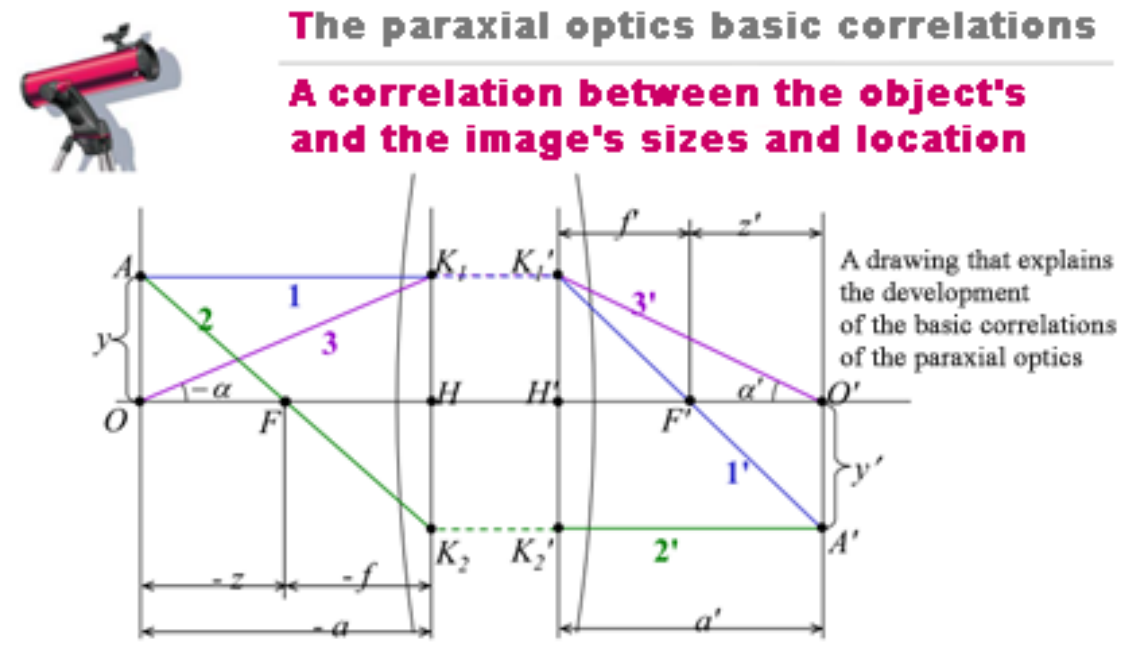

Fig.3 The example of the slide from the third part of the course "Geometrical Optics". Paraxial optics

The sixth part discusses the performance characteristics of optical systems, and one of the parts gives the main ideas about matrix optics and its applications for ideal optical system design and ray tracing.

The seventh part of the course is about the stops and pupils, about the limitations of ray tracing in real optical systems. It is usually one of the most difficult parts of such courses; it requires many examples and figures with step-by-step explanations. 
In the eighth part devoted to real optical systems we consider again the general principles of geometric optics, but for real surfaces. In other words, we consider here for what systems (surfaces) the Fermat's principle can be fulfilled, give these cases and present the examples of their using in real systems and optical devices.
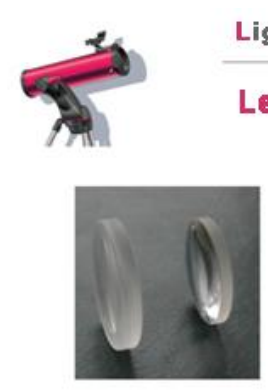

Lightguides and lenses

\section{Lenses}

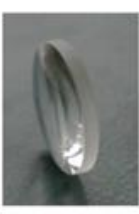

Lens is a component part made from

the transparent optical materials bordered by two high-polished

refracting surfaces

Properties:

-paraxial specification,

-aberrational

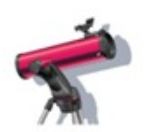

- Single Porro prism

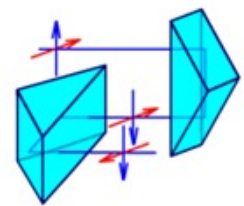

Plane mirrors and prlsms

Prism syatems

Fig.4 The example of the slides from the course "Geometrical Optics". Optical elements

In the last part we give the overview of simple optical components including lenses with spherical surfaces, some types of aspherical lenses, cylindrical lenses and their application, mirrors, plates and wedges, reflective prisms and optical fibers.

The main feature of the course concludes in involvement of young speakers among MS and PhD students of the Applied and computer Optics Department, ITMO University. This idea of the course authors is connected with perception features of undergraduates. If theory is demonstrated and explained by young people the virtual barrier of the unfathomable knowledge is removed. The young speakers also can make some changes to the course presentation, from the point of view of the young students that can adopt the material for the student's audience.

\section{TEST TASKS AND IMPLEMENTATION}

The theoretical part of the course presentation is more or less traditional, but one of the main difficulties for on-line courses is the learning outcomes evaluation. One of the possible variants is tests, where the student can choose the correct answer, or type the calculated value, or type the word or phrase.

For many courses these forms of test task is enough. Additional advantage of such form for this course is that we can use tasks that were checked, verified and adopted from other disciplines that deal with various topics of optics developed by the same authors. Additionally, such a situation speeds up the development of the course.

On the figures 5-6 the examples of the test tasks are shown.
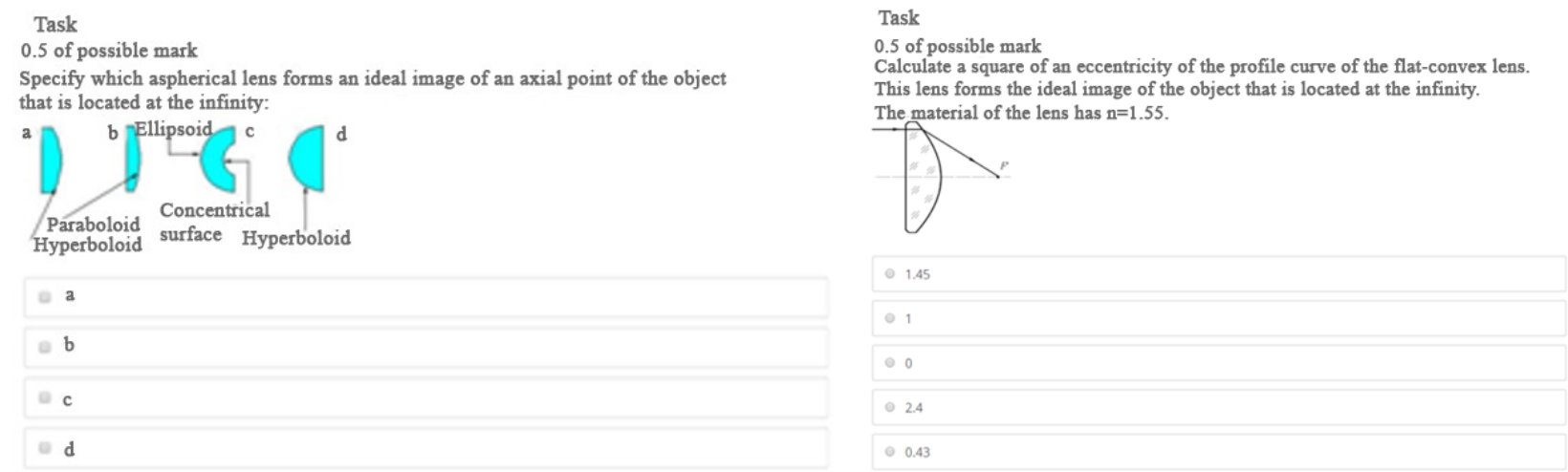

Fig.5 The example of the test task: questions about the lenses that give ideal image of the point source 


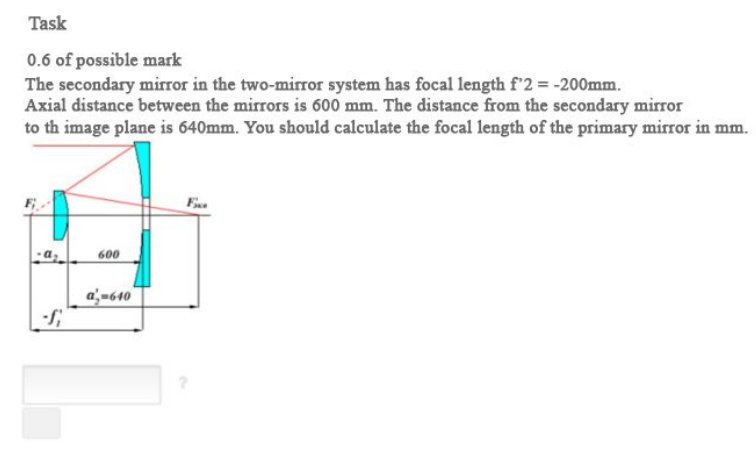

Fig.6 The example of the test task: questions about the mirror system

But for geometrical optics in some cases we should check the knowledge and skills in manual ray tracing. Usually this process even for simple ideal lenses leads to deeper understanding of the material. For this case a special computer tool was developed. This virtual lab is intended to estimate the understanding of the theory in the graphic ray tracing through simple ideal thin lenses.

The program generates a unique task for every student and can check if the answer is correct using only a few numbers. The task variants are generated such a way to be convenient to trace rays.

The program interface is given in a figure 7. Division of area of the screen into three fields of representation is provided in the program: margin of drawing, field of comments and field of buttons.

In the field of the drawing the carried-out graphical representation is displayed. In the field of comments, depending on the pressed key, the comments containing short instructions for the sequence of actions of the student, and also necessary instructions are output.

Comments represent presentation of the sequence of actions which are needed to adhere, and also several useful tips on use of this practical work.

The developed tool works in two modes: image construction and ray tracing.

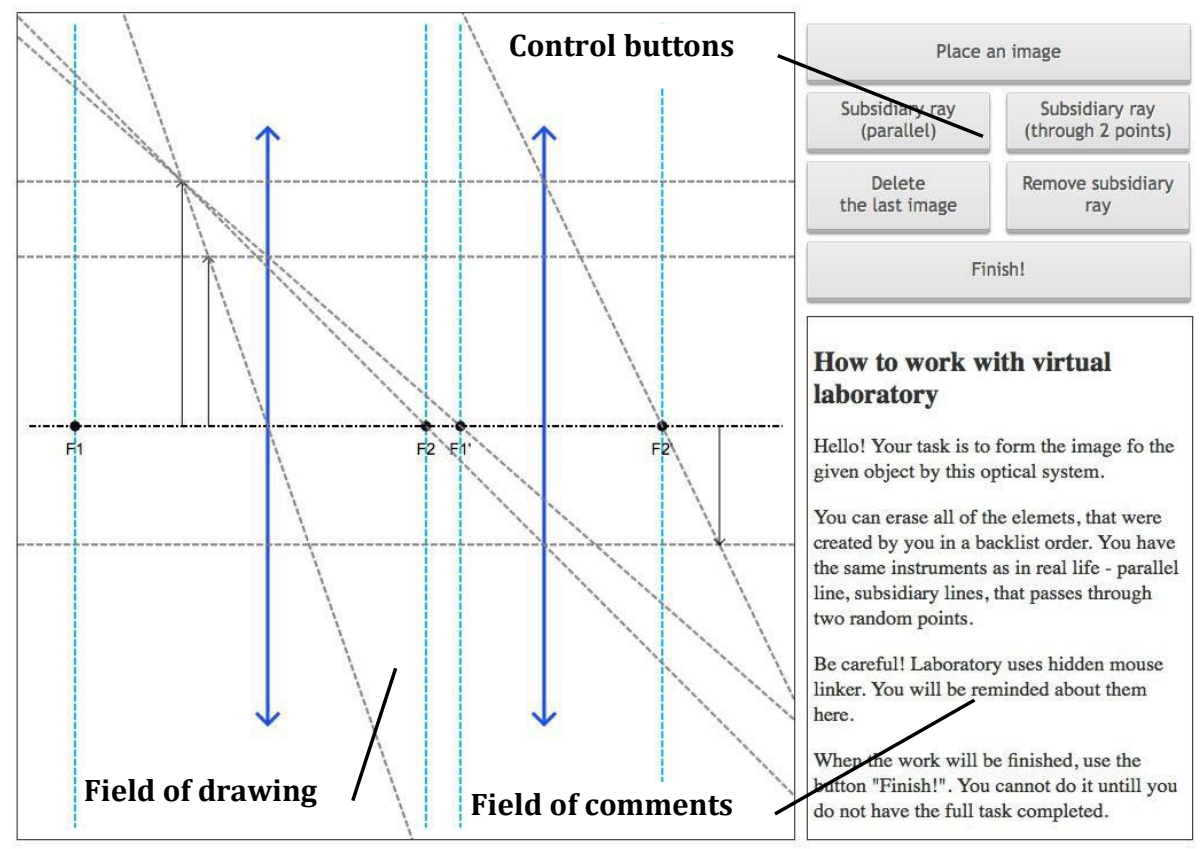

Fig. 7 Appearance of a virtual lab and methodical complex in the mode of an image construction 
In a figure 8 appearance of the program in the mode of ray tracing through an optical system is shown. Apparently, it differs in a little changed unit of buttons, and also the task.

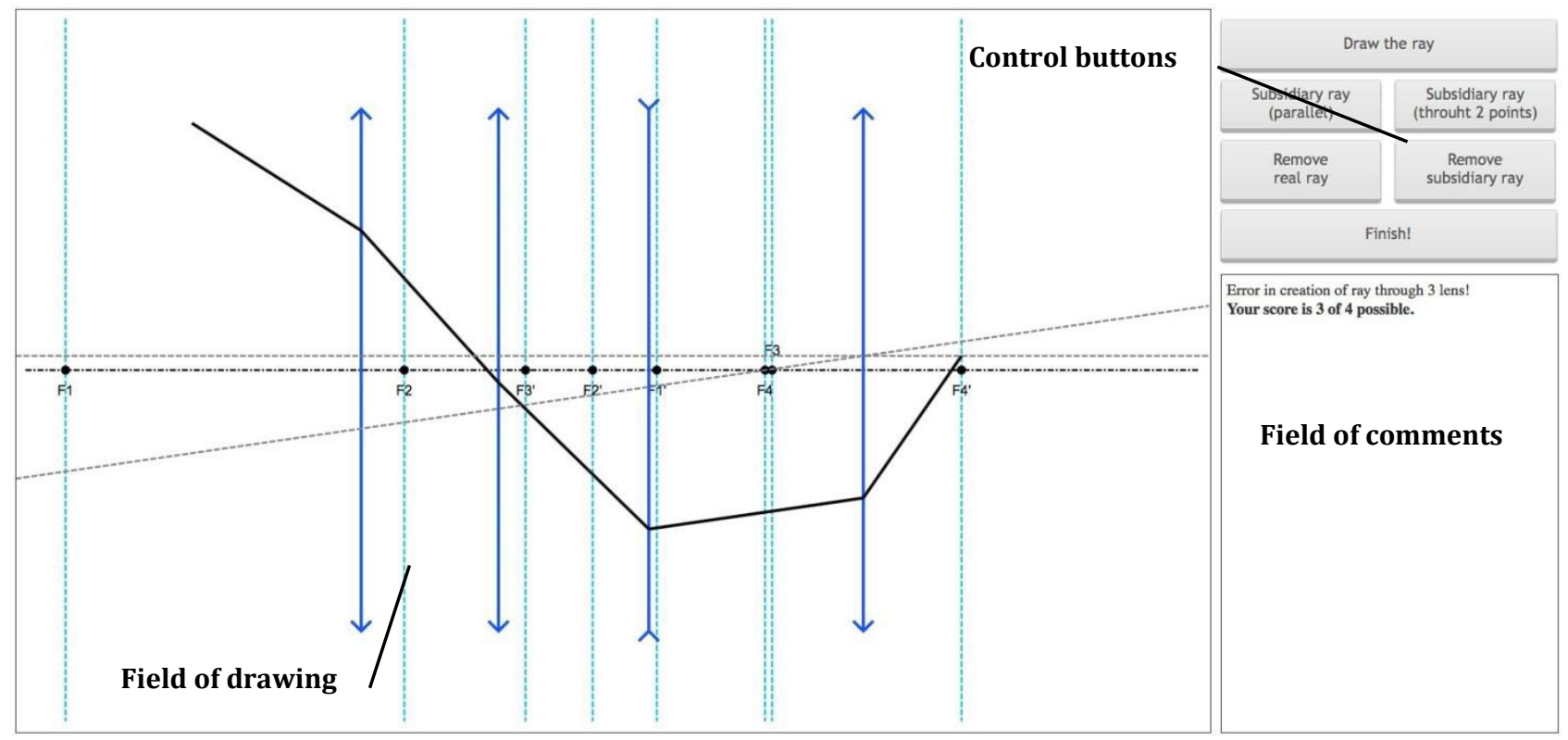

Fig. 8 Appearance of a computer laboratory and methodical complex in the mode of the ray trace construction

The developed program has some special features. As was mentioned earlier, the unique variants are generated for every student. If this process was random it would lead to some cases where ray construction was inconvenient, not visual and messy. To check if the generated variant is reasonable and realizable or not several simple equations are used derived for two cases: image construction mode and ray tracing mode.

For the ray tracing mode we use for the converging lens:

$$
\beta=\arccos \left[\frac{h+\frac{f}{\operatorname{tg} \alpha}}{\sqrt{\left(h+\frac{f}{\operatorname{tg} \alpha}\right)^{2}+f^{2}}}\right],
$$

for the diverging lens:

$$
\beta=180-\arccos \left[\frac{h+\frac{f}{\operatorname{tg} \alpha}}{\sqrt{\left(h+\frac{f}{\operatorname{tg} \alpha}\right)^{2}+f^{2}}}\right] .
$$

Here $\alpha$ - angle of incoming ray in degrees, $\beta$ - angle of outgoing ray in degrees, $h$ - height of incoming ray in pixels, $f=-f^{\prime}-$ front focus with sign.

It is possible to calculate $\beta$ if you know $\alpha, h, f=-f$ ' and compare it with user's value. In case of mouse linking on client side is used values can be checked with mistake $0.1 \%$.

An image position for current lens can also be calculated, because an image of previous lens is an object for the next lens, where $a^{\prime}$ - image position, $d$ - axial distance between components $i$ and $i-1$ :

$$
a_{i}=-\left(d-a_{i-1}^{\prime}\right)
$$

Image position can be calculated by a formula: 


$$
a^{\prime}=\frac{f^{\prime}}{1+\frac{f^{\prime}}{a}}
$$

Image size can be calculated by another formula:

$$
y^{\prime}=y *\left(\frac{f^{\prime}}{a+f^{\prime}}\right)
$$

If $f^{\prime}, a$ and $y$ are known, $a^{\prime}$ and $y^{\prime}$ can be calculated and compared with user's values. In case of mouse linking on client side is used values can also be checked with mistake $0.1 \%$.

\section{CONCLUSION}

The developed course is one of the parts of the national program in e-learning. As soon as the course was started in 2016 more than 500 students signed. It confirms that the optical engineering is urgently demanded professional area.

As all e-learning courses for the geometrical optics studies it has advantages and disadvantages, and of cause cannot replace intramural learning. But it can give a good basis for those students who want to study more advanced courses in the university and for those who want to know more about the optical phenomenon.

\section{ACKNOWLEDGEMENT}

This work was supported by the Ministry of Education and Science of Russian Federation (project №27.5615.2017/5.1).

\section{REFERENCES}

[1] http://en.ifmo.ru ITMO University

[2] http://cde.ifmo.ru Center of distance education, ITMO University

[3] http://openedu.ru Open education platform

[4] http://aco.ifmo.ru Applied and computer optics department, ITMO University 